\title{
STATIONARY SOLUTIONS FOR INTEGER-VALUED AUTOREGRESSIVE PROCESSES
}

\author{
EMAD-ELDIN A. A. ALY AND NADJIB BOUZAR
}

Received 5 January 2004 and in revised form 5 November 2004

The purpose of this paper is to introduce and develop a family of $\mathbb{Z}_{+}$-valued autoregressive processes of order $p(\operatorname{INAR}(p))$ by using the generalized multiplication $\odot_{F}$ of van Harn and Steutel (1982). We obtain various distributional and regression properties for these models. A number of stationary $\operatorname{INAR}(p)$ processes with specific marginals are presented and are shown to generalize several existing models.

\section{Introduction}

Integer-valued time series have been the object of growing interest in recent years. Models of stationary time series for count data with a given marginal distribution have been developed by several authors. Applications in the areas of model-fitting for count data and simulation of sequences of dependent Poisson and negative binomial (among others) variables have been offered. These models are based on the binomial thinning operator $\odot$ of Steutel and van Harn [19] which is defined as follows. If $X$ is a $\mathbb{Z}_{+}$-valued random variable (rv) and $\alpha \in(0,1)$, then

$$
\alpha \odot X=\sum_{i=1}^{X} X_{i},
$$

where $\left\{X_{i}\right\}$ is a sequence of i.i.d. Bernoulli $(\alpha)$ rv's independent of $X$. The operation $\odot$ incorporates the discrete nature of the variates and acts as the analogue of the standard multiplication used in the Box-Jenkins models. For example, an integer-valued first-order autoregressive (INAR(1)) process is described by the following equation:

$$
X_{n+1}=\alpha \odot X_{n-1}+\epsilon_{n} .
$$

McKenzie $[14,16]$ used the binomial thinning operator to construct stationary Poisson and negative binomial autoregressive moving average (namely, $\operatorname{AR}(1), \operatorname{MA}(q)$, and $\operatorname{ARMA}(1, q))$ processes. Al-Osh and Alzaid [1] offered a general theory for integer-valued moving average processes of order $q(\operatorname{INMA}(q))$. Du and Li [7] (see also Jayakumar [10] and Latour [11]) developed higher-order integer-valued autoregressive processes of order 
$p(\operatorname{INAR}(p))$ processes. Related models that made use of a more general operator were introduced by Aly and Bouzar [2] and Zhu and Joe [22]. We refer to McKenzie [17] for an overview of the work in this area (and for further references).

van Harn et al. [21] introduced the generalized multiplication $\odot_{F}$ (see definition below) as an extension of binomial thinning and used it to define concepts of discrete selfdecomposability and stability. Subsequently, van Harn and Steutel [20] used $\odot_{F}$ to define and solve stability equations involving continuous-time $\mathbb{Z}_{+}$-valued processes with stationary independent increments.

The purpose of this paper is to introduce and develop a family of $\operatorname{INAR}(p)$ processes by using the $\odot_{F}$ multiplication in lieu of binomial thinning see Definitions 2.1 and 5.1. We obtain various distributional and regression properties for these models. A number of stationary $\operatorname{INAR}(p)$ processes with specific marginals are presented and are shown to generalize several existing models. The paper is organized as follows. In Section 2, we introduce the F-INAR(1) process and give several properties. Stationary solutions for FINAR(1) processes and characterizations of their marginals are offered in Section 3. In Section 4, we study the question of time-reversibility for a stationary F-INAR(1) process and we obtain a form for the probability generating function (pgf) of its marginal. Section 5 is devoted to higher-order $F-\operatorname{INAR}(p)$ models and their properties.

In the rest of this section, we recall some definitions and results that are needed in the sequel. For proofs and further details, we refer to Athreya and Ney (see [5, Chapter 3]), van Harn et al. [21], and van Harn and Steutel [20]. The pgf of a distribution $\left(p_{n}, n \geq 0\right)$ on $\mathbb{Z}_{+}$is defined by

$$
P(z)=\sum_{n=0}^{\infty} p_{n} z^{n} \quad(|z| \leq 1) .
$$

$F:=\left(F_{t}, t \geq 0\right)$ will denote a continuous composition semigroup of pgf's such that $F_{t} \not \equiv 1$ and $\delta_{F}=-\ln F_{1}^{\prime}(1)>0$. For any $|z| \leq 1$,

$$
F_{s} \circ F_{t}(z)=F_{s+t}(z) \quad(s, t \geq 0), \quad \lim _{t \downarrow 0} F_{t}(z)=z, \quad \lim _{t \rightarrow \infty} F_{t}(z)=1
$$

The infinitesimal generator $U$ of the semigroup $F$ is defined by

$$
U(z)=\lim _{t \downarrow 0} \frac{F_{t}(z)-z}{t} \quad(|z| \leq 1)
$$

and satisfies $U(z)>0$ for $0 \leq z<1$. There exist a constant $a>0$ and a distribution $\left(h_{n}, n \geq\right.$ $0)$ on $\mathbb{Z}_{+}$with pgf $H(z)$ such that $h_{1}=0$,

$$
\begin{gathered}
H^{\prime}(1)=\sum_{n=1}^{\infty} n h_{n} \leq 1, \\
U(z)=a\{H(z)-z\}, \quad|z| \leq 1 .
\end{gathered}
$$


The related $A$-function is defined by

$$
A(z)=\exp \left\{-\int_{0}^{z}(U(x))^{-1} d x\right\}, \quad z \in[0,1)
$$

The functions $U(z)$ and $A(z)$ satisfy

$$
U\left(F_{t}(z)\right)=U(z) F_{t}^{\prime}(z), \quad A\left(F_{t}(z)\right)=e^{-t} A(z) \quad(t \geq 0,0 \leq z \leq 1) .
$$

Moreover,

$$
\delta_{F}=a\left(1-H^{\prime}(1)\right)=-U^{\prime}(1), \quad F_{t}^{\prime}(1)=e^{-\delta_{F} t} \quad(t \geq 0) .
$$

The function $B(z)$ defined by

$$
B(z)=\lim _{t \rightarrow \infty} \frac{F_{t}(z)-F_{t}(0)}{1-F_{t}(0)}
$$

is a pgf such that $B(0)=0$ and takes the form

$$
B(z)=1-A(z)^{\delta_{F}}
$$

For a $\mathbb{Z}_{+}$-valued $\operatorname{rv} X$ and $\eta \in(0,1)$, the generalized multiplication $\eta \odot_{F} X$ is defined by

$$
\eta \odot_{F} X=\sum_{i=1}^{X} Y_{i},
$$

where $\left(Y_{i}, i \geq 1\right)$ is a sequence of i.i.d. rv's independent of $X$ with common $\operatorname{pgf} F_{t}, t=$ $-\ln \eta$.

Throughout the paper, stationarity of a stochastic process is considered to be in the strict sense. Finally, $P_{X}$ will denote the pgf of the distribution of a $\mathbb{Z}_{+}$-valued $\operatorname{rv} X$.

\section{F-INAR(1) processes}

Definition 2.1. A sequence $\left(X_{n}, n \in \mathbb{Z}\right)$ of $\mathbb{Z}_{+}$-valued rv's is said to be an $F$-INAR(1) process if for any $n \in \mathbb{Z}$,

$$
X_{n}=\eta \odot_{F} X_{n-1}+\epsilon_{n}
$$

where $0<\eta<1$ and $\left(\epsilon_{n}, n \in \mathbb{Z}\right)$ is an i.i.d. sequence of $\mathbb{Z}_{+}$-valued rv's that is assumed to be independent of the $Y$ variables that define the operator $\odot_{F}$ (see below). $\left(\epsilon_{n}, n \in \mathbb{Z}\right)$ is called the innovation sequence.

In the remainder of this paper, we will at times refer to the single-ended $F$-INAR(1) process $\left(X_{n}, n \geq 0\right)$ that arises when (2.1) is assumed to hold only for $n \geq 0$. 
The generalized multiplication $\eta \odot_{F} X_{n-1}$ in (2.1) is performed independently for each $n$. More precisely, we assume the existence of an array $\left(Y_{i, n}, i \geq 0, n \in \mathbb{Z}\right)$ of i.i.d. $\mathbb{Z}_{+}-$ valued rv's, independent of $\left(\epsilon_{n}, n \in \mathbb{Z}\right)$, such that the array's common pgf is $F_{t}(z), t=$ $-\ln \eta$, and (see (1.13))

$$
\eta \odot_{F} X_{n-1}=\sum_{i=1}^{X_{n-1}} Y_{i, n-1} .
$$

These assumptions clearly make the model (2.1) a Markov chain.

The pgf's $P_{X_{n}}(z)$ and $P_{\epsilon}(z)$ of the $F$-INAR(1) process (2.1) satisfy the equation

$$
P_{X_{n}}(z)=P_{X_{n-1}}\left(F_{t}(z)\right) P_{\epsilon}(z), \quad t=-\ln \eta, n \in \mathbb{Z} .
$$

By using (2.3) recursively (and the fact that $F_{t}(z)$ is a semigroup), it can be shown that an $F$-INAR(1) process $\left(X_{n}, n \in \mathbb{Z}\right)$ admits the following representation for any $k \geq 1$ :

$$
X_{n} \stackrel{d}{=} \eta^{k} \odot_{F} X_{n-k}+\sum_{i=0}^{k-1} \eta^{i} \odot_{F} \epsilon_{n-i}, \quad n \in \mathbb{Z} .
$$

Further distributional and correlation properties of $F$-INAR(1) processes are gathered in the following proposition.

Proposition 2.2. Assume $\sum_{n=2}^{\infty} n(n-1) h_{n}<\infty$. Let $\left(X_{n}, n \in \mathbb{Z}\right)$ be an F-INAR(1) process (for some $0<\eta<1$ ) such that $E\left(X_{n}\right)<\infty$ and $E\left(X_{n}^{2}\right)<\infty$ for any $n \in \mathbb{Z}, \mu_{\epsilon}=E\left(\epsilon_{0}\right)<\infty$ and $\sigma_{\epsilon}^{2}=\operatorname{Var}\left(\epsilon_{0}\right)<\infty$.

(i) The regression of $X_{n}$ on $X_{n-1}$ is linear:

$$
E\left(X_{n} \mid X_{n-1}\right)=\eta^{\delta_{F}} X_{n-1}+\mu_{\epsilon}, \quad n \in \mathbb{Z} .
$$

(ii) The conditional variance of $X_{n}$ given $X_{n-1}$ is linear:

$$
\operatorname{Var}\left(X_{n} \mid X_{n-1}\right)=A X_{n-1}+\sigma_{\epsilon}^{2}, \quad n \in \mathbb{Z}
$$

where $A=\left(1-U^{\prime \prime}(1) / U^{\prime}(1)\right) \eta^{\delta_{F}}\left(1-\eta^{\delta_{F}}\right)$.

(iii) For any $n \in \mathbb{Z}$ and $k \geq 0$, the covariance at lag $k, \Gamma_{n}(k)=\operatorname{cov}\left(X_{n-k}, X_{n}\right)$ of $\left\{X_{n}\right\}$, is

$$
\Gamma_{n}(k)=\eta^{k \delta_{F}} \operatorname{Var}\left(X_{n-k}\right) .
$$

(iv) For any $n \in \mathbb{Z}$ and $k \geq 0$,

$$
\begin{gathered}
E\left(X_{n}\right)=\eta^{k \delta_{F}} E\left(X_{n-k}\right)+\mu_{\epsilon} \sum_{i=0}^{k-1} \eta^{i \delta_{F}} \\
\operatorname{Var}\left(X_{n}\right)=\eta^{2 k \delta_{F}} \operatorname{Var}\left(X_{n-k}\right)+A \sum_{i=1}^{k} \eta^{2(i-1) \delta_{F}} E\left(X_{n-i}\right)+\sigma_{\epsilon}^{2} \sum_{i=1}^{k} \eta^{2(i-1) \delta_{F}}
\end{gathered}
$$

where $A$ is as in (2.6) above. 
Proof. First, we note (by (1.7)) that $\sum_{n=2}^{\infty} n(n-1) h_{n}<\infty$ implies that $U^{\prime \prime}(1)$ exists. By (2.2) and (1.10), $E\left(\eta \odot_{F} X_{n-1} \mid X_{n-1}\right)=\eta^{\delta_{F}} X_{n-1}$ which leads to (2.5). By differentiating twice (with respect to $z$ ) the expression $U\left(F_{t}(z)\right)=F_{t}^{\prime}(z) U(z)(t=-\ln \eta)$ and letting $z \rightarrow 1$, we obtain $F_{t}^{\prime \prime}(1)=\eta^{\delta_{F}}\left(\eta^{\delta_{F}}-1\right) U^{\prime \prime}(1) / U^{\prime}(1)$. Again, by $(2.2)$ and $(1.10), E\left(\left(\eta \odot_{F}\right.\right.$ $\left.\left.X_{n-1}\right)^{2} \mid X_{n-1}\right)=\operatorname{Var}\left(Y_{1, n-1}\right) X+\eta^{\delta_{F}} X^{2}$. Noting that

$$
\operatorname{Var}\left(Y_{1, n-1}\right)=F_{t}^{\prime \prime}(1)+F_{t}^{\prime}(1)-F_{t}^{\prime}(1)^{2}=\eta^{\delta_{F}}\left(1-\eta^{\delta_{F}}\right)\left(1-\frac{U^{\prime \prime}(1)}{U^{\prime}(1)}\right)
$$

(2.6) follows by direct calculations. Equation (2.7) is obtained by applying a conditioning argument to (2.4). Finally, (2.8) are easily derived from (2.5) and (2.6).

The following result due to Latour [11] insures the existence of a stationary F-INAR(1) process.

Proposition 2.3. Given $0<\eta<1$ and a sequence $\left(\epsilon_{n}, n \in \mathbb{Z}\right)$ of i.i.d. $\mathbb{Z}_{+}$-valued rv's with finite mean $\mu_{\epsilon}$ and finite variance $\sigma_{\epsilon}^{2}$, there exists a stationary F-INAR(1) process $\left(X_{n}, n \in\right.$ $\mathbb{Z})$ satisfying (2.1) and such that $\operatorname{cov}\left(X_{m}, \epsilon_{n}\right)=0, m<n$.

Next, we explore the relationship between discrete self-decomposability and stationary $F$-INAR(1) processes. A distribution on $\mathbb{Z}_{+}$with $\operatorname{pgf} P(z)$ is said to be $F$-self-decomposable (van Harn et al. [21]) if for any $t>0$, there exists a pgf $P_{t}(z)$ such that

$$
P(z)=P\left(F_{t}(z)\right) P_{t}(z), \quad|z| \leq 1 .
$$

Any $F$-self-decomposable distribution can arise as the marginal distribution of a stationary F-INAR(1) process. More precisely, we have the following result.

Proposition 2.4. Let $P(z)$ be the pgf of an F-self-decomposable distribution. For any $\eta \in$ $(0,1)$, there exists a stationary $F$-INAR(1) process $\left(X_{n}, n \in \mathbb{Z}\right)$ whose marginal distribution has $\operatorname{pg} f(z)$.

Proof. Using (2.10), one can construct for every $\eta \in(0,1)$, a single-ended $F$-INAR(1) process $\left(X_{n}, n \geq 0\right)$ of the form (2.1) whose innovation sequence $\left(\epsilon_{n}, n \geq 0\right)$ has common $\operatorname{pgf} P_{t}(z)$ (where $t=-\ln \eta$ ) and such that $X_{0}$ has pgf $P(z)$. It follows from (2.3) and (2.10) that the $X_{n}$ 's are identically distributed, which implies that $\left(X_{n}, n \geq 0\right)$ is stationary since it is a Markov chain. The double-ended version is obtained by sliding $\left(X_{n}, n \geq 0\right)$ to the left.

Next, we state a representation theorem for stationary F-INAR(1) processes. The proof follows easily from (2.4) and is omitted.

Proposition 2.5. Any stationary F-INAR(1) process $\left(X_{n}, n \in \mathbb{Z}\right)$ admits the following (infinite-order) moving average representation for some $0<\eta<1$ :

$$
X_{n} \stackrel{d}{=} \sum_{i=0}^{\infty} \eta^{i} \odot_{F} \epsilon_{n-i}, \quad n \in \mathbb{Z},
$$

where the convergence of the series is in the weak sense. 
The mean, variance, and autocorrelation function (ACRF) of a stationary F-INAR(1) process follow straightforwardly from Proposition 2.2.

Corollary 2.6. Assume $\sum_{n=2}^{\infty} n(n-1) h_{n}<\infty$. Let $\left(X_{n}, n \in \mathbb{Z}\right)$ be a stationary F-INAR(1) process (for some $0<\eta<1$ ) such that $E\left(X_{0}\right)<\infty, E\left(X_{0}^{2}\right)<\infty, \mu_{\epsilon}=E\left(\epsilon_{0}\right)<\infty$, and $\sigma_{\epsilon}^{2}=$ $\operatorname{Var}\left(\epsilon_{0}\right)<\infty$. Then

(i) for any $n \in \mathbb{Z}$,

$$
\begin{gathered}
E\left(X_{n}\right)=\mu_{\epsilon}\left(1-\eta^{\delta_{F}}\right)^{-1}, \\
\operatorname{Var}\left(X_{n}\right)=\frac{\left(1-U^{\prime \prime}(1) / U^{\prime}(1)\right) \eta^{\delta_{F}} \mu_{\epsilon}+\sigma_{\epsilon}^{2}}{1-\eta^{2 \delta_{F}}} ;
\end{gathered}
$$

(ii) for any $k \geq 0$ and $n \in \mathbb{Z}$, the correlation coefficient of $\left(X_{n-k}, X_{n}\right)$ is

$$
\rho(k)=\eta^{k \delta_{F}}
$$

We note that the ACRF of a stationary F-INAR(1) process, as given by (2.13), has the same form as that of the standard $\mathrm{AR}(1)$ process. It decays exponentially at lag $k$. However, unlike the standard $\operatorname{AR}(1)$ case, $\rho(k)$ remains always positive.

Remark 2.7. Assume that $\left(X_{n}, n \geq 0\right)$ is a one-sided $F$-INAR(1) process. If the pgf's $P_{\epsilon}(z)$ and $F_{t}(z)(t=-\ln \eta)$ satisfy

$$
\int_{0}^{1} \frac{1-P_{\epsilon}(x)}{F_{t}(x)-x} d x<\infty, \quad t=-\ln \eta,
$$

then by Foster and Williamson [9], $\left(X_{n}, n \geq 0\right)$ admits a limiting distribution. Endowing $X_{0}$ with the limiting distribution leads to stationarity (since $\left(X_{n}, n \geq 0\right)$ is a Markov chain).

\section{Stationary solutions of $F$-INAR(1) processes}

In this section, we present several stationary solutions of $F$-INAR(1) processes.

A $\mathbb{Z}_{+}$-valued rv $X$ is said to have an $F$-stable distribution with exponent $\gamma>0$ if there exists a sequence of i.i.d. rv's $\left(X_{i}, i \geq 0\right), X_{i} \stackrel{d}{=} X$ for all $i$, such that for any $n>0, X \stackrel{d}{=}$ $n^{-1 / \gamma} \odot_{F} \sum_{i=1}^{n} X_{i}$ (see van Harn et al. [21]). F-stable distributions are $F$-self-decomposable and exist only when $0<\gamma \leq \delta_{F}$. Moreover, the $\operatorname{pgf} P(z)$ of an $F$-stable distribution with exponent $\gamma \in\left(0, \delta_{F}\right]$ admits the canonical representation

$$
P(z)=\exp \left[-\lambda A(z)^{\gamma}\right]
$$

for some $\lambda>0$, where $A(z)$ is given in (1.8).

It follows by Proposition 2.4 that for every $0<\eta<1$, there exists a stationary $F$ $\operatorname{INAR}(1)$ process $\left(X_{n}, n \in \mathbb{Z}\right)$ with an $F$-stable marginal distribution with exponent $\gamma$ $\left(0<\gamma \leq \delta_{F}\right)$. The marginal distribution of the innovation sequence $\left(\epsilon_{n}, n \in \mathbb{Z}\right)$, obtained by solving for $P_{\epsilon}$ in (2.3) and by using (1.9), is also $F$-stable with exponent $\gamma$ and has pgf

$$
P_{\epsilon}(z)=\exp \left[-\lambda\left(1-\eta^{\gamma}\right) A(z)^{\gamma}\right]
$$


Moreover, it can be shown (see van Harn et al. [21]) that stationary F-INAR(1) processes whose marginal is $F$-stable with finite mean arise only in the case $\gamma=\delta_{F}$ and $B^{\prime}(1)<\infty$ (where $B(z)$ is given by (1.12)). The process has finite variance if $B^{\prime \prime}(1)<\infty$.

We have shown above (by letting $\eta=e^{-t}$ ) that the $\operatorname{pgf} P(z)$ of the marginal distribution of a stationary $F$-stable $F$-INAR(1) process satisfies the following property. For any $t>0$, there exist $c(t) \in(0,1)$ such that

$$
P\left[F_{t}(z)\right]=P(z)^{c(t)}, \quad 0 \leq z \leq 1 .
$$

It turns out that this property characterizes such processes.

Proposition 3.1. Let $P(z)$ be a pgf such that $P(z) \neq 0$ for all $0 \leq z \leq 1$. Then $P(z)$ is $F$ stable, with some exponent $\gamma \in\left(0, \delta_{F}\right]$, if and only if for any $t>0$, there exists $c(t) \in(0,1)$ such that (3.3) holds. The function $c(t)$ is necessarily of the form $c(t)=e^{-\gamma t}$.

Proof. We need only to prove the "if" part. Let $\psi(z)=\ln P(z)$. It follows by (3.3) that for any $t>0$, there exists $c(t) \in(0,1)$ such that

$$
\psi\left(F_{t}(z)\right)=c(t) \psi(z)
$$

Letting $\psi_{1}(z)=\psi(z) / \psi(0)$ and noting that $c(t)=\psi\left(F_{t}(0)\right) / \psi(0),(3.4)$ becomes

$$
\psi_{1}\left(F_{t}(z)\right)=\psi_{1}\left(F_{t}(0)\right) \psi_{1}(z) .
$$

By differentiating (3.5) with respect to $t$, we obtain

$$
\frac{\partial}{\partial t} F_{t}(z) \psi_{1}^{\prime}\left(F_{t}(z)\right)=\frac{\partial}{\partial t} F_{t}(0) \psi_{1}^{\prime}\left(F_{t}(0)\right) \psi_{1}(z)
$$

Using $(\partial / \partial t) F_{t}(z)=U\left(F_{t}(z)\right)$ and letting $t \downarrow 0$, it follows by (1.4) that

$$
\frac{\psi_{1}^{\prime}(z)}{\psi_{1}(z)}=\frac{U(0)}{U(z)} \psi_{1}^{\prime}(0),
$$

whose solution is $\psi_{1}(z)=A(z)^{\gamma}$, where $\gamma=-\psi_{1}^{\prime}(0) U(0)>0$. Hence, $P(z)$ has the form (3.1). Since $P$ is a pgf, $\gamma$ must satisfy $\gamma \leq \delta_{F}$ (cf. van Harn and Steutel [20, the proof of Lemma 4.2]). The form of $c(t)$ follows from its uniqueness and the "only if" part.

Next, we present a stationary $F$-INAR(1) process with an $F$-geometric stable marginal distribution.

A $\mathbb{Z}_{+}$-valued $\mathrm{rv} X$ is said to have an $F$-geometric stable distribution if for any $p \in(0,1)$, there exists $\alpha(p) \in(0,1)$ such that $X \stackrel{d}{=} \alpha(p) \odot_{F} \sum_{i=1}^{N_{p}} X_{i}$, where $\left(X_{i}, i \geq 1\right)$ is a sequence of i.i.d. rv's, $X_{i} \stackrel{d}{=} X, N_{p}$ has the geometric distribution with parameter $p$, and $\left(X_{i}, i \geq 1\right)$ and $N_{p}$ are independent (see Bouzar [6]). F-geometric stable distributions are $F$-selfdecomposable and their pgf's admit the canonical representation

$$
P(z)=\left(1+\lambda A(z)^{\gamma}\right)^{-1}
$$


for $0<\gamma \leq \delta_{F}$ and $\lambda>0$. We will refer to distributions with pgf (3.8) as $F$-geometric stable distributions with exponent $\gamma$.

By Proposition 2.4, there exists, for every $\eta \in(0,1)$, a stationary $F$-INAR(1) process $\left(X_{n}, n \in \mathbb{Z}\right)$ with an $F$-geometric stable marginal distribution with pgf (3.8). Its innovation sequence $\left(\epsilon_{n}, n \in \mathbb{Z}\right)$ has marginal pgf (obtained by solving (2.3) for $P_{\epsilon}(z)$ and by using (1.9))

$$
P_{\epsilon}(z)=\eta^{\gamma}+\left(1-\eta^{\gamma}\right)\left(1+\lambda A(z)^{\gamma}\right)^{-1}, \quad 0<\gamma \leq \delta_{F}, \lambda>0
$$

This implies that a stationary $F$-INAR(1) process $\left(X_{n}, n \in \mathbb{Z}\right)$ with an $F$-geometric stable distribution can be written as

$$
X_{n}=\eta \odot_{F} X_{n-1}+I_{n} E_{n}, \quad n \in \mathbb{Z},
$$

where $\left(I_{n}, n \in \mathbb{Z}\right)$ and $\left(E_{n}, n \in \mathbb{Z}\right)$ are independent sequences of i.i.d. rv's such that $I_{n}$ is Bernoulli $\left(1-\eta^{\gamma}\right)$ and $E_{n}$ has the same distribution as $X_{n}$. Moreover, a stationary $F$ INAR(1) process with an $F$-geometric stable marginal has finite mean only if $\gamma=\delta_{F}$ and $B^{\prime}(1)<\infty$. It has a finite variance if $B^{\prime \prime}(1)<\infty$.

We have in fact shown by the above argument (and by letting $\eta=e^{-t}$ ) that the pgf $P(z)$ of the marginal distribution of a stationary $F$-geometric stable $F$-INAR $(1)$ process satisfies the following property. For any $t>0$, there exists $c(t) \in(0,1)$ such that

$$
P(z)=P\left(F_{t}(z)\right)(c(t)+(1-c(t)) P(z)) .
$$

We show next that the converse is true.

Proposition 3.2. Let $P(z)$ be the pgf of a nondegenerate distribution on $\mathbb{Z}_{+}$. Then $P(z)$ is $F$-geometric stable with some exponent $\gamma \in\left(0, \delta_{F}\right]$ if and only if for any $t>0$, there exists $c(t) \in(0,1)$ such that (3.11) holds. The function $c(t)$ is necessarily of the form $c(t)=e^{-\gamma t}$.

Proof. We only need to show the "if" part. Rewriting $P(z)=(1+\psi(z))^{-1}$, it follows by (3.11) that for $t>0$, there exists $c(t) \in(0,1)$ such that $\psi\left(F_{t}(z)\right)=c(t) \psi(z)$. Using the exact same argument as the one in the proof of Proposition 3.1 (following (3.5)), we have $\psi(z)=\lambda A(z)^{\gamma}$ for some $0<\gamma \leq \delta_{F}$ and $\lambda>0$. The form of $c(t)$ follows from its uniqueness and the "only if" part.

We define next a compound discrete Linnik distribution and construct the corresponding stationary $F$-INAR(1) process.

$\mathrm{A} \mathbb{Z}_{+}$-valued rv $X$ is said to have an $F$-compound discrete Linnik distribution if its pgf has the form

$$
P(z)=\left(1+\lambda A(z)^{\gamma}\right)^{-r}
$$

for some $0<\gamma \leq \delta_{F}, \lambda>0$, and $r>0$. Note that $P(z)$ indeed results from the compounding of i.i.d. rv's (with the common pgf $B(z)$ of (1.12)) by a discrete Linnik distribution (with pgf $\left.G(z)=\left(1+\lambda(1-z)^{\gamma / \delta_{F}}\right)^{-r}\right)$. The special case $r=1$ in (3.12) gives the $F$-geometric stable distribution. van Harn and Steutel [20] showed that F-compound discrete Linnik 
distributions are $F$-self-decomposable and arise as solutions to stability equations for $\mathbb{Z}_{+}-$ valued processes with stationary independent increments.

Again by self-decomposability, for every $\eta \in(0,1)$, there exists a stationary $F$-INAR(1) process $\left(X_{n}, n \in \mathbb{Z}\right)$ with an $F$-compound Linnik marginal distribution (with pgf (3.12)). Its innovation sequence $\left\{\epsilon_{n}\right\}$ has pgf

$$
P_{\epsilon}(z)=\left(\frac{1+\lambda \eta^{\gamma} A(z)^{\gamma}}{1+\lambda A(z)^{\gamma}}\right)^{r}
$$

It can be shown by a straightforward pgf argument that $\left\{\epsilon_{n}\right\}$ has the representation

$$
\epsilon \stackrel{d}{=} \sum_{i=1}^{N}\left(\eta^{U_{i}}\right) \odot_{F} W_{i}
$$

where $\left(W_{i}, i \geq 0\right)$ is a sequence of i.i.d. F-geometric stable rv's (with pgf (3.8)), $\left\{U_{i}\right\}$ are i.i.d. uniform $(0,1)$ rv's, and $N$ is Poisson with mean $-r \gamma \ln \eta$, with all these variables independent. This allows for a shot-noise interpretation of the process that is similar to the one given by Lawrance [12] for the gamma AR(1) process (see also McKenzie [15] for the case of the negative binomial INAR(1) process). A shot-noise process is defined by

$$
X(t)=\sum_{m=N(-\infty)}^{N(t)} \eta^{t-\tau_{m}} \odot W_{m}
$$

where $\left(W_{m}, m \geq 0\right)$ are $\mathbb{Z}_{+}$-valued i.i.d. rv's (amplitudes of the shots) and $(N(t), t \geq 0)$ is a Poisson process with occurrence times at $\tau_{m}$. If the $W_{m}$ 's have their common pgf given by (3.8) and $N(t)$ has rate $-r \gamma \ln \eta$, then $X(t)$ of (3.15), sampled at $n=0, \pm 1, \pm 2, \ldots$ gives another representation of the stationary $F$-INAR(1) process (2.1) with marginal pgf (3.12). The proof of this fact is an adaptation of Lawrance's [12] argument and the details are omitted.

Finally, as above, a stationary $F$-INAR(1) process with an $F$-compound discrete Linnik marginal has finite mean only if $\gamma=\delta_{F}$ and $B^{\prime}(1)<\infty$. It has a finite variance if $B^{\prime \prime}(1)<\infty$.

van Harn et al. [21] give some rich examples of continuous composition semigroups of pgf's from which one can generate $F$-INAR(1) processes. We mention the parametrized family of semigroups $\left(F^{(\theta)}, \theta \in[0,1)\right)$ described by

$$
F_{t}^{(\theta)}(z)=1-\frac{\bar{\theta} e^{-\bar{\theta} t}(1-z)}{\bar{\theta}+\theta\left(1-e^{-\bar{\theta} t}\right)(1-z)}, \quad t \geq 0,|z| \leq 1, \bar{\theta}=1-\theta .
$$

In this case, we have $\delta_{F^{(\theta)}}=\bar{\theta}, U_{F^{(\theta)}}(z)=(1-z)(1-\theta z)$ and $A_{F^{(\theta)}}(z)=((1-z) /(1-$ $\theta z))^{1 / \bar{\theta}}$. We note that for $\theta=0, F^{(\theta)}$ corresponds to the standard semigroup $F_{t}^{(0)}(z)=$ $1-e^{-t}+e^{-t} z$ and the multiplication $\odot_{F^{(0)}}$ becomes the binomial thinning operator of Steutel and van Harn [19].

The Poisson AR(1) process of McKenzie [16] is the stationary $F^{(0)}$-INAR(1) process with an $F^{(0)}$-stable marginal. More generally, the Poisson geometric INAR(1) process of 
Aly and Bouzar [2] arises as the stationary $F^{(\theta)}$-INAR(1) process with an $F^{(\theta)}$-stable marginal. The discrete Mittag-Leffler $F$-INAR(1) process of Pillai and Jayakumar [10] (and, in particular, the geometric INAR(1) of McKenzie [14]) is the stationary $F^{(0)} \operatorname{INAR}(1)$ process with an $F^{(0)}$-geometric stable marginal. The discrete Linnik INAR(1) process of Aly and Bouzar [3] (and, in particular, the negative binomial INAR(1) of McKenzie [14]) is the stationary $F^{(0)}$-INAR(1) process with an $F^{(0)}$-compound Linnik marginal.

Finally, we note that Zhu and Joe [22] used a reparametrized version of the semigroup $F^{(\theta)}$ to construct a continuous-time $\mathbb{Z}_{+}$-valued Markov process $(X(t), t \geq 0)$ via the equation

$$
X(t)=e^{-\mu(t-s)} \odot_{F^{(\theta)}} X(s)+\epsilon(s, t), \quad s<t,
$$

where $\mu>0$ and $\epsilon(s, t)$ is $\mathbb{Z}_{+}$-valued and independent of $X(s)$.

\section{Time-reversibility of stationary $F$-INAR(1) processes}

A stochastic process $\left(X_{n}, n \in \mathbb{Z}\right)$ is said to be time-reversible if for any $n \in \mathbb{Z}$ and $k \geq 0$, $\left(X_{n}, X_{n+1}, \ldots, X_{n+k}\right)$ and $\left(X_{n+k}, X_{n+k-1}, \ldots, X_{n}\right)$ have the same joint distribution.

Let $\left(X_{n}, n \in \mathbb{Z}\right)$ be an $F$-INAR(1) process. By the Markov property, $\left(X_{n}, n \in \mathbb{Z}\right)$ is time-reversible if and only if for any $n \in \mathbb{Z},\left(X_{n-1}, X_{n}\right)$ and $\left(X_{n}, X_{n-1}\right)$ have the same joint distribution. In terms of the joint $\operatorname{pgf} g_{n}\left(z_{1}, z_{2}\right)$ of $\left(X_{n-1}, X_{n}\right)$ which is defined by

$$
g_{n}\left(z_{1}, z_{2}\right)=E\left(z_{1}^{X_{n-1}} z_{2}^{X_{n}}\right) \quad\left(\left|z_{1}\right| \leq 1,\left|z_{2}\right| \leq 1\right),
$$

$\left(X_{n}, n \in \mathbb{Z}\right)$ is time-reversible if and only if $g_{n}\left(z_{1}, z_{2}\right)=g_{n}\left(z_{2}, z_{1}\right)$ for all $n \in \mathbb{Z},\left|z_{1}\right| \leq 1$, and $\left|z_{2}\right| \leq 1$.

By (2.1) and a conditioning argument, it is easily shown that

$$
g_{n}\left(z_{1}, z_{2}\right)=P_{\epsilon}\left(z_{2}\right) P_{X_{n-1}}\left(z_{1} F_{t}\left(z_{2}\right)\right) \quad(t=-\ln \eta, 0<\eta<1) .
$$

By Proposition 2.2(i), a time-reversible $F$-INAR(1) process $\left(X_{n}, n \in \mathbb{Z}\right.$ ) (such that $E\left(X_{n}\right)<\infty$ and $\left.E\left(\epsilon_{n}\right)<\infty\right)$ possesses the property of linear backward regression. That is, there exist $c>0$ and $d \geq 0$ such that for any $n \in \mathbb{Z}$,

$$
E\left(X_{n-1} \mid X_{n}\right)=d+c X_{n}
$$

We show next that under an $x \ln x$ condition (condition (4.4) below), a stationary $F$ INAR(1) process with finite mean and finite variance has the property of backward linear regression only if its pgf admits a certain form.

Proposition 4.1. Assume that the distribution $\left(h_{n}, n \geq 0\right)$ satisfies

$$
\sum_{n=2}^{\infty} h_{n} n \ln n<\infty .
$$

Let $\left(X_{n}, n \in \mathbb{Z}\right)$ be a stationary F-INAR(1) process with finite mean and finite variance with the property of linear backward regression (4.3). Then the pgf $P(z)$ of the marginal 
distribution of $\left(X_{n}, n \in \mathbb{Z}\right)$ has the form

$$
P(z)=\exp \left(-\lambda \int_{z}^{1} \frac{B(x)}{x} d x\right)
$$

where $\lambda>0$ and $B(z)$ is the pgf of (1.12).

Proof. Let $n \geq 1$ and let $g\left(z_{1}, z_{2}\right)$ be the joint pgf of $\left(X_{n-1}, X_{n}\right)$. We have by (2.3) and (4.2)

$$
g\left(z_{1}, z_{2}\right)=\frac{P\left(z_{1} F_{t}\left(z_{2}\right)\right) P\left(z_{2}\right)}{P\left(F_{t}\left(z_{2}\right)\right)} \quad(t=-\ln \eta, 0<\eta<1) .
$$

Differentiating $g$ with respect to $z_{1}$, then setting $z_{1}=1$ and $z_{2}=z$, it follows that for any $n \in \mathbb{Z}$,

$$
E\left(X_{n-1} z^{X_{n}}\right)=\frac{F_{t}(z) P(z) P^{\prime}\left(F_{t}(z)\right)}{P\left(F_{t}(z)\right)} .
$$

Now by (4.3) we have for some $c>0$ and $d \geq 0$,

$$
E\left(X_{n-1} z^{X_{n}}\right)=E\left(z^{X_{n}} E\left(X_{n-1} \mid X_{n}\right)\right)=c z E\left(X_{n} z^{X_{n}-1}\right)+d E\left(z^{X_{n}}\right)
$$

for any $n \geq 1$. Letting $Q(z)=z P^{\prime}(z) / P(z)$ and combining (4.7) and (4.8) (note that $\left.E\left(X_{n} z^{X_{n}-1}\right)=P^{\prime}(z)\right)$, we obtain $c Q(z)+d=Q\left(F_{t}(z)\right)$, and therefore, by differentiation, $c Q^{\prime}(z)=F_{t}^{\prime}(z) Q^{\prime}\left(F_{t}(z)\right)$. Noting that $Q^{\prime}(1)=\operatorname{Var}\left(X_{n}\right) \neq 0$, it follows that $c=F_{t}^{\prime}(1)=$ $e^{-\delta_{F} t}$ (with the second equation following from (1.10)) which implies

$$
Q^{\prime}(z)=e^{\delta_{F} t} F_{t}^{\prime}(z) Q^{\prime}\left(F_{t}(z)\right)
$$

An induction argument yields for any $n \geq 1$,

$$
Q^{\prime}(z)=e^{n \delta_{F} t} \prod_{j=0}^{n-1} F_{t}^{\prime}\left(F_{j t}(t)\right) Q^{\prime}\left(F_{n t}(z)\right) .
$$

By the semigroup property and (1.9), we have $F_{t}^{\prime}\left(F_{j t}(z)\right)=U\left(F_{(j+1) t}(z)\right) / U\left(F_{j t}(z)\right), j=$ $0, \ldots, n-1$. Therefore,

$$
Q^{\prime}(z)=e^{n \delta_{F} t} \frac{U\left(F_{n t}(z)\right)}{U(z)} Q^{\prime}\left(F_{n t}(z)\right)
$$

From the semigroup properties (1.4), (1.10), and (1.11), we have

$$
\lim _{n \rightarrow \infty} F_{n t}(z)=1, \quad \lim _{n \rightarrow \infty} \frac{U\left(F_{n t}(z)\right)}{F_{n t}(z)-1}=U^{\prime}(1)=-\delta_{F}, \quad \lim _{n \rightarrow \infty} \frac{F_{n t}(z)-1}{F_{n t}(0)-1}=1-B(z) .
$$

Moreover, (4.4) implies (see van Harn et al. [21])

$$
\lim _{n \rightarrow \infty} e^{n \delta_{F} t}\left(F_{n t}(0)-1\right)=-1 \text {. }
$$


Therefore, by letting $n \rightarrow \infty$ in (4.11), we obtain

$$
Q^{\prime}(z)=\delta_{F} Q^{\prime}(1) \frac{1-B(z)}{U(z)}
$$

Since (by (1.8) and (1.12)) $1 / U(z)=-A^{\prime}(z) / A(z)$ and $1-B(z)=A(z)^{\delta_{F}}$, we have (note that $Q(0)=0)$

$$
Q(z)=\int_{0}^{z} Q^{\prime}(x) d x=Q^{\prime}(1)\left(1-A(z)^{\delta_{F}}\right)=Q^{\prime}(1) B(z)
$$

which implies that $P^{\prime}(z) / P(z)=Q^{\prime}(1) B(z) / z$ or

$$
\ln P(z)=-Q^{\prime}(1) \int_{z}^{1} \frac{B(x)}{x} d x .
$$

We note that Proposition 4.1 remains valid if the property of backward linear regression is replaced by the (stronger) assumption of time-reversibility.

For the family of semigroups $\left(F^{(\theta)}, \theta \in[0,1)\right)$ of (3.16), the condition (4.4) is satisfied ( since $h_{n}=0$ for $n \geq 3$ ). In this case, the $\operatorname{pgf} P(z)$ of (4.5) is shown to be

$$
P(z)= \begin{cases}e^{-\lambda(1-z)} & \text { if } \theta=0(\lambda>0), \\ \left(\frac{\bar{\theta}}{1-\theta z}\right)^{r} & \text { if } 0<\theta<1(r>0) .\end{cases}
$$

The Poisson distribution (resp., the negative binomial distribution with probability of success $\theta, 0<\theta<1)$ is the only distribution that arises as the marginal of a stationary $F^{(0)}-\operatorname{INAR}(1)$ (resp., $F^{(\theta)}$-INAR(1)) process with finite mean and finite variance and with the property of backward linear regression. These results were established by Alzaid and Al-Osh [4] (for $\theta=0$ ) and by Aly and Bouzar [2] (for $0<\theta<1$ ).

\section{An $F$-INAR $(p)$ process}

Lawrance and Lewis [13] introduced the mixed autoregressive process of order $p(\operatorname{AR}(p))$

$$
X_{n}=\sum_{i=1}^{p} I\left(\xi_{n}=i\right) \eta_{i} X_{n-i}+\epsilon_{n}
$$

where $I(A)$ is the indicator function of the event $A,\left\{\xi_{n}\right\}$ and $\left\{\epsilon_{n}\right\}$ are two independent sequences of i.i.d. rv's, $0<\eta_{i}<1, P\left(\xi_{n}=i\right)=c_{i}, i=1,2, \ldots, p$, and $\sum_{i=1}^{p} c_{i}=1$. The authors obtained the distribution of the innovation $\mathrm{rv} \epsilon_{n}$ for the stationary $\operatorname{AR}(p)$ process with an exponential marginal. Pillai and Jayakumar [18] went a bit further by deriving the distribution of $\epsilon_{n}$ for the stationary $\operatorname{AR}(p)$ process with the Mittag-Leffler marginal on $\mathbb{R}_{+}$. Using the binomial thinning operator of Steutel and van Harn [19], Jayakumar [10] defined the discrete analogue of (5.1) and constructed the discrete Mittag-Leffler $\operatorname{INAR}(p)$ process. 
In this section, we present a generalized $\operatorname{INAR}(p)$ process by using the $\odot_{F}$ operator. In particular, we will derive the marginal distribution of the stationary $\operatorname{INAR}(p)$ process with an $\mathrm{F}$-geometric stable marginal.

Definition 5.1. A sequence $\left\{X_{n}\right\}$ of $\mathbb{Z}_{+}$-valued rv's is said to be an $F$-INAR $(p)$ process if for any $n \in \mathbb{Z}$,

$$
X_{n}=\sum_{i=1}^{p} I\left(\xi_{n}=i\right) \eta_{i} \odot_{F} X_{n-i}+\epsilon_{n}
$$

where $\left(\xi_{n}, n \in \mathbb{Z}\right)$ and $\left(\epsilon_{n}, n \in \mathbb{Z}\right)$ are two independent sequences of i.i.d. $\mathbb{Z}_{+}$-valued rv's, $0<\eta_{i}<1, P\left(\xi_{n}=i\right)=c_{i}, i=1,2, \ldots, p$, and $\sum_{i=1}^{p} c_{i}=1$.

The generalized multiplication $\eta_{i} \odot_{F} X_{n-i}$ in (5.2) is performed independently for each $i$. More precisely, we assume the existence of $p$ independent arrays $\left(Y_{i, n}^{(j)}, i \geq 0, n \in\right.$ $\mathbb{Z}), j=1,2, \ldots, p$, of i.i.d. $\mathbb{Z}_{+}$-valued rv's, independent of $\left(\xi_{n}, n \in \mathbb{Z}\right)$ and $\left(\epsilon_{n}, n \in \mathbb{Z}\right)$, such that for each $j=1,2, \ldots, p$, the array's common $\operatorname{pgf}$ is $F_{t_{j}}(z), t_{j}=-\ln \eta_{j}$, and

$$
\eta_{j} \odot_{F} X_{n-j}=\sum_{i=1}^{X_{n-j}} Y_{i, n-j}^{(j)}
$$

In terms of pgf's, it follows from (5.2) that

$$
P_{X_{n}}(z)=\left(\sum_{i=1}^{p} c_{i} P_{X_{n-i}}\left(F_{t_{i}}(z)\right)\right) P_{\epsilon}(z), \quad t_{i}=-\ln \eta_{i} .
$$

The autocorrelation structure of a stationary $F-\operatorname{INAR}(p)$ process is given in the following proposition and its corollary.

Proposition 5.2. Assume $\sum_{n=2}^{\infty} n(n-1) h_{n}<\infty$. Let $\left(X_{n}, n \in \mathbb{Z}\right)$ be a stationary $F$ $\operatorname{INAR}(p)$ process such that $E\left(X_{0}\right)<\infty, E\left(X_{0}^{2}\right)<\infty, \mu_{\epsilon}=E\left(\epsilon_{0}\right)<\infty$, and $\sigma_{\epsilon}^{2}=\operatorname{Var}\left(\epsilon_{0}\right)<\infty$. Let $c_{i}$ and $\eta_{i}(i=1, \ldots, p)$ be as in (5.2). Then

(i) for any $n \in \mathbb{Z}$,

$$
E\left(X_{n}\right)=\left(1-\sum_{i=1}^{p} c_{i} \eta_{i}^{\delta_{F}}\right)^{-1} \mu_{\epsilon}
$$

(ii) the autocovariance function $\Gamma(k)=\operatorname{Cov}\left(X_{n-k}, X_{n}\right)$ of $\left(X_{n}, n \in \mathbb{Z}\right)$ is given by

$$
\Gamma(k)= \begin{cases}\sum_{i=1}^{p} c_{i} \eta_{i}^{\delta_{F}} \Gamma(i)+B & \text { if } k=0, \\ \sum_{i=1}^{p} c_{i} \eta_{i}^{\delta_{F}} \Gamma(k-i) & \text { if } k \geq 1,\end{cases}
$$


with $\Gamma(k)=\Gamma(-k), k \geq 1$, and

$$
B=\sigma_{\epsilon}^{2}+\frac{1-U^{\prime \prime}(1)}{U^{\prime}(1)} E\left(X_{0}\right) \sum_{i=1}^{p} c_{i} \eta_{i}^{\delta_{F}}\left(1-\eta_{i}^{\delta_{F}}\right)+E\left(X_{0}^{2}\right) \sum_{i=1}^{p}\left(c_{i}-c_{i}^{2}\right) \eta_{i}^{2 \delta_{F}}
$$

Proof. Stationarity implies $\Gamma(k)=\Gamma(-k)$. Using (5.2), (1.10), and a conditioning argument yields (5.5) and

$$
E\left(X_{n-k}\left(\eta_{i} \odot_{F} X_{n-i}\right)\right)=\eta_{i}^{\delta_{F}} E\left(X_{n-k} X_{n-i}\right) \quad(i=1,2, \ldots, p) .
$$

It follows that $E\left(X_{n} X_{n-k}\right)=\sum_{i=1}^{p} c_{i} \eta_{i}^{\delta_{F}} E\left(X_{n-k} X_{n-i}\right)+E\left(\epsilon_{n}\right) E\left(X_{n-k}\right)$. Combining this last equation with (5.5) gives the formula for $\Gamma(k), k \geq 1$, in (5.6). Direct calculations lead to (note again that $U^{\prime \prime}(1)$ exists because $\sum_{n=2}^{\infty} n(n-1) h_{n}<\infty$ )

$$
\Gamma(0)=\sum_{i=1}^{p} c_{i}^{2} \eta_{i}^{2 \delta_{F}} \Gamma(0)+2 \sum_{1 \leq i<j \leq p} c_{i} c_{j} \eta_{i}^{\delta_{F}} \eta_{j}^{\delta_{F}} \Gamma(j-i)+B
$$

where $B$ is as in (5.7). This in turn leads to $\Gamma(0)$ of (5.6).

The sequence $(\Gamma(k), k \in \mathbb{Z})$ is completely determined by (5.6) once $\Gamma(k), k=0, \ldots, p$, are found. The latter are solution of the linear system (5.6) restricted to $k=0,1, \ldots, p$. Since $\sum_{i=1}^{p} c_{i} \eta_{i}^{\delta_{F}}<1$, it follows by Latour [11, Lemma 2.1] that this system has a unique solution given by

$$
\Gamma_{\mathbf{p}}=B(\mathbf{I}-\mathbf{C})^{-1} \mathbf{c},
$$

where $\boldsymbol{\Gamma}_{\mathbf{p}}=(\Gamma(0), \Gamma(1), \ldots, \Gamma(p)), \mathbf{I}$ is the $(p+1) \times(p+1)$ identity matrix, $\mathbf{C}$ is also $(p+$ $1) \times(p+1)$ whose $(i, j)$ entry is equal to the coefficient of $\Gamma(i)$ on the right-hand side of the $j$ th equation of $(5.6), i, j=0,1, \ldots, p, \mathbf{c}=(1,0, \ldots, 0)$ (of length $p+1$ ), and $B$ is given by (5.7).

Corollary 5.3. Assume $\sum_{n=2}^{\infty} n(n-1) h_{n}<\infty$. Let $\left(X_{n}, n \in \mathbb{Z}\right)$ be a stationary F-INAR $(p)$ process such that $E\left(X_{0}\right)<\infty, E\left(X_{0}^{2}\right)<\infty, \mu_{\epsilon}=E\left(\epsilon_{0}\right)<\infty$ and $\sigma_{\epsilon}^{2}=\operatorname{Var}\left(\epsilon_{0}\right)<\infty$. Then the autocorrelation coefficient $\rho_{k}$ at lag $k, k \geq 1$, is given by

$$
\rho_{k}=\sum_{i=1}^{p} c_{i} \eta_{i}^{\delta_{F}} \rho_{k-i}
$$

where $\rho_{0}=1, \rho_{k}=\rho_{-k}$, and $c_{i}$ and $\eta_{i}(i=1, \ldots, p)$ are as in (5.2).

Next, we determine the marginal distribution of the innovation sequence of a stationary $F$-INAR $(p)$ process with an $F$-geometric stable marginal distribution (whose pgf is given by (3.8)). We need a lemma first. 
Lemma 5.4. For $i=1,2$, let $f_{i}(t)=\sum_{j=1}^{n_{i}} c_{i j}\left(a_{i j} /\left(a_{i j}+t\right)\right), t \geq 0, a_{i j}>0, c_{i j} \geq 0$, and $\sum_{j=1}^{n_{i}} c_{i j}$ $=1$. If $\max _{1 \leq i \leq n_{1}} a_{1 i}<\min _{1 \leq i \leq n_{2}} a_{2 i}$, there exist $c_{k}^{\prime} \geq 0, d_{k}>0$, with $\sum_{k=0}^{n_{1}+n_{2}-1} c_{k}^{\prime}=1$ and $d_{k}$ strictly increasing (with the $d_{k}$ 's being the $a_{1 k}$ 's in ascending order for $k=1, \ldots, n_{1}$ ) such that

$$
\frac{f_{1}(t)}{f_{2}(t)}=c_{0}^{\prime}+\sum_{k=1}^{n_{1}+n_{2}-1} c_{k}^{\prime} \frac{d_{k}}{d_{k}+t}
$$

Proof. Note that $f_{i}(t)$ is the Laplace-Stieltjes transform of a mixture of exponential distributions. By grouping and reordering if necessary, we may assume without loss of generality that $0<a_{i j}<\cdots<a_{i n_{i}}, i=1,2$. Using a result in Feller [8, pages 438-439], we have

$$
f_{i}(t)=C_{i} \cdot \frac{b_{i 1}+t}{a_{i 1}+t} \cdots \cdots \frac{b_{i, n_{i}-1}+t}{a_{i, n_{i}-1}+t} \cdot \frac{1}{a_{i n_{i}}+t}
$$

where $0<a_{i 1}<b_{i 1}<a_{i 2}<b_{i 2}<\cdots<b_{i, n_{i}-1}<a_{i n_{i}}$ and $C_{i}>0$. Letting $d_{k}=a_{1 k}, k=1, \ldots$, $n_{1}$, and $d_{n_{1}+k}=b_{2 k}, k=1, \ldots, n_{2}-1$, a straightforward partial fraction expansion leads to (5.12). The fact that $a_{1 n_{1}}<a_{21}$ and $f_{1}(0) / f_{2}(0)=1$ implies that $c_{k}^{\prime} \geq 0, \sum_{k=0}^{n_{1}+n_{2}-1} c_{k}^{\prime}=1$, and $d_{k}$ is increasing by definition.

Proposition 5.5. If $\left(X_{n}, n \in \mathbb{Z}\right)$ is a stationary $F$-INAR $(p)$ process with an F-geometric stable marginal (with pgf given by (3.8)), then

$$
\epsilon_{n} \stackrel{d}{=} \sum_{j=0}^{p} I\left(V_{n}=j\right) \beta_{j} \odot_{F} E_{n}
$$

where $\left(V_{n}, n \in \mathbb{Z}\right)$ and $\left(E_{n}, n \in \mathbb{Z}\right)$ are independent sequences of i.i.d. $r v$ 's, $P\left(V_{n}=j\right)=c_{j}^{\prime}$, $j=0, \ldots, p, \sum_{j=0}^{p} c_{j}^{\prime}=1, E_{n}$ has the same distribution as $X_{n}, \beta_{0}=0, \beta_{1}=1$, and $0<\beta_{i}<1$ for $i=2, \ldots, p$.

Proof. Using (1.9), (3.8), and (5.4), we have

$$
P_{\epsilon}(z)=\frac{\left(1+\lambda A(z)^{\gamma}\right)^{-1}}{\sum_{i=1}^{p} c_{i} a_{i}\left(a_{i}+\lambda A(z)^{\gamma}\right)^{-1}},
$$

with $\lambda>0,0<\gamma \leq \delta_{F}$, and $a_{i}=\eta_{i}^{-\gamma}>1, i=1, \ldots, p$. By applying Lemma 5.4 to (5.15) with $n_{1}=1, n_{2}=p, a_{11}=1, a_{2 j}=a_{j}$, and $t=\lambda A(z)^{\gamma}$, we obtain

$$
P_{\epsilon}(z)=c_{0}^{\prime}+c_{1}^{\prime}\left(1+\lambda A(z)^{\gamma}\right)^{-1}+\sum_{j=2}^{p} c_{j}^{\prime}\left(1+\lambda \beta_{j}^{\gamma} A(z)^{\gamma}\right)^{-1}
$$

with $\beta_{j}^{\gamma}=d_{j}^{-1}, d_{j}>1$ (by Lemma 5.4), for $j=2, \ldots, p$. The representation (5.14) then follows easily from (5.16). 
The proof of Proposition 5.5 shows that in the case of a stationary $F$-INAR $(p)$ process with an $F$-geometric stable marginal, the solution (5.16) of (5.4) exists for the full range of $p,\left\{t_{i}\right\}$, and $\left\{c_{i}\right\}$. It turns out that the converse is true. That is, if $P(z)$ is the pgf of a nondegenerate distribution such that (5.16) is the solution of (5.4) (where $P_{X_{k}}(z)=P(z)$ for all $k$ 's) for the full range of $p,\left\{t_{i}\right\}$, and $\left\{c_{i}\right\}$, then $P(z)$ is necessarily $F$-geometric stable. This is a direct consequence of Proposition 3.2, since, applied to the case $p=1$, the assumption reduces to (3.11).

Finite mixtures of $F$-geometric stable distributions can arise as marginals of stationary $F-\operatorname{INAR}(p)$ processes. Such distributions have a pgf of the form

$$
P(z)=\sum_{j=1}^{m} q_{j}\left(1+\lambda_{j} A(z)^{\gamma}\right)^{-1},
$$

where $0<\gamma \leq \delta_{F}, \lambda_{j}>0, q_{j} \geq 0$, and $\sum_{j=1}^{m} q_{j}=1$. Solving (5.4) for $P_{\epsilon}$, with $P_{X}$ as in (5.17), gives

$$
P_{\epsilon}(z)=\frac{\sum_{j=1}^{m} q_{j} b_{j}\left(b_{j}+A(z)^{\gamma}\right)^{-1}}{\sum_{i=1}^{p} \sum_{j=1}^{m} c_{i} q_{j} b_{i j}\left(b_{i j}+A(z)^{\gamma}\right)^{-1}},
$$

where $b_{j}=1 / \lambda_{j}$ and $b_{i j}=b_{j} \eta_{i}^{-\gamma}$. If

$$
\frac{\max _{1 \leq i \leq m} \lambda_{i}}{\min _{1 \leq i \leq m} \lambda_{i}}<\min _{1 \leq i \leq p} \eta_{i}^{-\gamma}
$$

then a straightforward application of Lemma $5.4\left(n_{1}=m, n_{2}=p\right.$, the $a_{1 k}$ 's are the $b_{k}$ 's, the $a_{2 k}$ 's are the $b_{i j}$ 's, and $\left.t=A(z)^{\gamma}\right)$ shows that $P_{\epsilon}(z)$ is the pgf of a mixture of $F$-geometric stable distributions and the degenerate distribution at 0 .

One can use the family of semigroups $\left(F^{(\theta)}, \theta \in[0,1)\right)$ of (3.16) to construct stationary $F$-INAR $(p)$ processes with $F$-geometric stable (or finite mixtures of $F$-geometric stable) marginals. We note that Jayakumar's [10] discrete Mittag-Leffler $\operatorname{INAR}(p)$ process is the $F^{(0)}-\operatorname{INAR}(p)$ process with an $F^{(0)}$-geometric stable marginal. As seen above, finite mixtures of discrete Mittag-Leffler distributions can arise as the marginal of a stationary $F^{(0)}$-INAR $(p)$ process (under condition (5.19)). This is a counterexample to Jayakumar's [10, Theorem 2.2] that states that only discrete Mittag-Leffler distributions can arise as marginals of stationary $\operatorname{INAR}(p)$ processes.

Finally, we mention that a more general $F-\operatorname{INAR}(p)$ process can be constructed using Latour's [11] definition of an $\operatorname{INAR}(p)$ process. In this case, (5.2) becomes

$$
X_{n}=\sum_{i=1}^{p} \eta_{i} \odot_{F} X_{n-i}+\epsilon_{n},
$$

where $0<\eta_{i}<1, i=1, \ldots, p$, and $\left(\epsilon_{n}, n \in \mathbb{Z}\right)$ is an i.i.d. sequence of $\mathbb{Z}_{+}$-valued rv's with finite mean $\mu_{\epsilon}$ and finite variance $\sigma_{\epsilon}^{2}$. By Latour [11], a finite mean stationary $\operatorname{INAR}(p)$ 
process of type (5.20) exists if and only if

$$
\sum_{i=1}^{p} \eta_{i}^{\delta_{F}}<1
$$

Proposition 5.2 and Corollary 5.3 remain valid with the following modified formulas for the mean:

$$
E\left(X_{n}\right)=\left(1-\sum_{i=1}^{p} \eta_{i}^{\delta_{F}}\right)^{-1} \mu_{\epsilon}
$$

the autocovariance function (with $B_{1}=\sigma_{\epsilon}^{2}+\left(1-U^{\prime \prime}(1) / U^{\prime}(1)\right) E\left(X_{0}\right) \sum_{i=1}^{p} \eta_{i}^{\delta_{F}}\left(1-\eta_{i}^{\delta_{F}}\right)$ )

$$
\Gamma(k)= \begin{cases}\sum_{i=1}^{p} \eta_{i}^{\delta_{F}} \Gamma(i)+B_{1} & \text { if } k=0, \\ \sum_{i=1}^{p} \eta_{i}^{\delta_{F}} \Gamma(k-i) & \text { if } k \geq 1,\end{cases}
$$

and the autocorrelation coefficient

$$
\rho_{k}=\sum_{i=1}^{p} \eta_{i}^{\delta_{F}} \rho_{k-i}
$$

\section{Acknowledgment}

The authors wish to thank the referees for their comments which significantly improved the presentation of the paper.

\section{References}

[1] M. Al-Osh and A. A. Alzaid, Integer-valued moving average (INMA) process, Statist. Hefte 29 (1988), no. 4, 281-300.

[2] E.-E. Aly and N. Bouzar, Explicit stationary distributions for some Galton-Watson processes with immigration, Comm. Statist. Stochastic Models 10 (1994), no. 2, 499-517.

[3] _ On geometric infinite divisibility and stability, Ann. Inst. Statist. Math. 52 (2000), no. 4, 790-799.

[4] A. A. Alzaid and M. Al-Osh, First-order integer-valued autoregressive (INAR (1)) process: distributional and regression properties, Statist. Neerlandica 42 (1988), no. 1, 53-61.

[5] K. B. Athreya and P. E. Ney, Branching Processes, Springer-Verlag, New York, 1972.

[6] N. Bouzar, On geometric stability and Poisson mixtures, Illinois J. Math. 43 (1999), no. 3, 520527.

[7] J. G. Du and Y. Li, The integer-valued autoregressive (INAR $(p))$ model, J. Time Ser. Anal. 12 (1991), no. 2, 129-142.

[8] W. Feller, An Introduction to Probability Theory and Its Applications. Vol. II., John Wiley \& Sons, New York, 1971.

[9] J. H. Foster and J. A. Williamson, Limit theorems for the Galton-Watson process with timedependent immigration, Z. Wahrsch. Verw. Gebiete 20 (1971), 227-235. 
[10] K. Jayakumar, An integer valued autoregressive (INAR(p)) time series model, Bulletin of the International Statistical Institute, Proceedings of the 50th Session Book 3, 1995, pp. 1365-1374.

[11] A. Latour, Existence and stochastic structure of a non-negative integer-valued autoregressive process, J. Time Ser. Anal. 19 (1998), no. 4, 439-455.

[12] A. J. Lawrance, The innovation distribution of a gamma distributed autoregressive process, Scand. J. Statist. 9 (1982), 234-236.

[13] A. J. Lawrance and P. A. W. Lewis, The exponential autoregressive-moving average $\operatorname{EARMA}(p, q)$ process, J. Roy. Statist. Soc. Ser. B 42 (1980), no. 2, 150-161.

[14] E. McKenzie, Autoregressive moving-average processes with negative-binomial and geometric marginal distributions, Adv. in Appl. Probab. 18 (1986), no. 3, 679-705.

[15] - Innovation distributions for gamma and negative binomial autoregressions, Scand. J. Statist. 14 (1987), no. 1, 79-85.

[16] Some ARMA models for dependent sequences of Poisson counts, Adv. in Appl. Probab. 20 (1988), no. 4, 822-835.

[17] _ Discrete variate time series, Stochastic Processes: Modelling and Simulation (D. N. Shanbhag and C. R. Rao, eds.), Handbook of Statist., vol. 21, North-Holland, Amsterdam, 2003, pp. 573-606.

[18] R. N. Pillai and K. Jayakumar, Specialised class L property and stationary autoregressive process, Statist. Probab. Lett. 19 (1994), no. 1, 51-56.

[19] F. W. Steutel and K. van Harn, Discrete analogues of self-decomposability and stability, Ann. Probab. 7 (1979), no. 5, 893-899.

[20] K. van Harn and F. W. Steutel, Stability equations for processes with stationary independent increments using branching processes and Poisson mixtures, Stochastic Process. Appl. 45 (1993), no. 2, 209-230.

[21] K. van Harn, F. W. Steutel, and W. Vervaat, Self-decomposable discrete distributions and branching processes, Z. Wahrsch. Verw. Gebiete 61 (1982), no. 1, 97-118.

[22] R. Zhu and H. Joe, A new type of discrete self-decomposability and its application to continuoustime Markov processes for modeling count data time series, Stoch. Models 19 (2003), no. 2, 235-254.

Emad-Eldin A. A. Aly: Department of Statistics and Operations Research, Kuwait University, P.O. Box 5969, Safat 13060, Kuwait

E-mail address: emad@kuc01.kuniv.edu.kw

Nadjib Bouzar: Department of Mathematics and Computer Science, University of Indianapolis, Indianapolis, IN 46227, USA

E-mail address: nbouzar@uindy.edu 


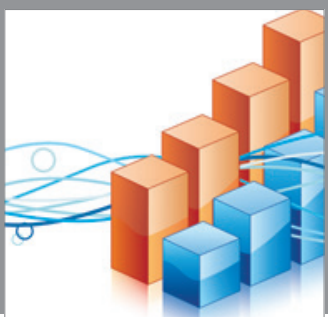

Advances in

Operations Research

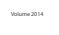

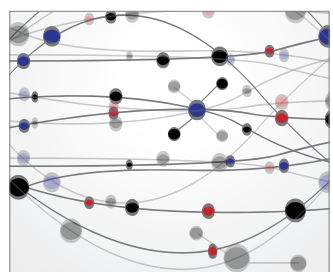

\section{The Scientific} World Journal
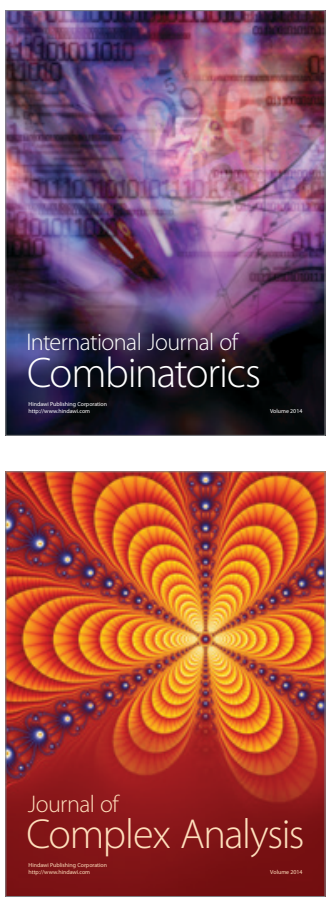

International Journal of

Mathematics and

Mathematical

Sciences
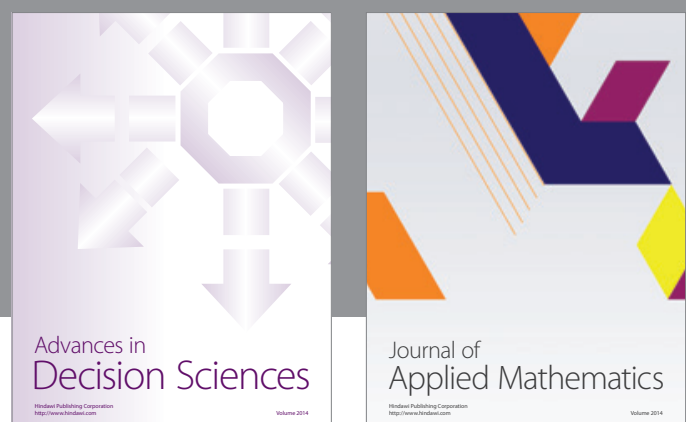

Journal of

Applied Mathematics
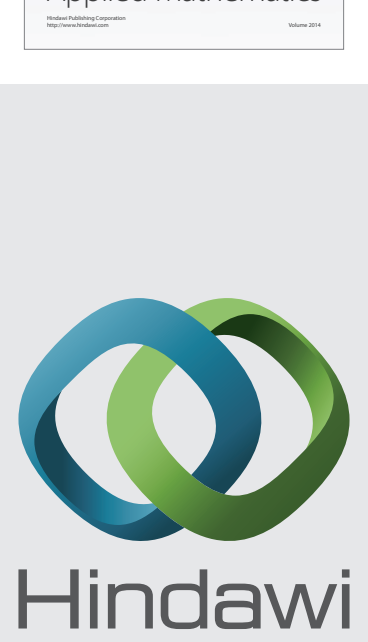

Submit your manuscripts at http://www.hindawi.com
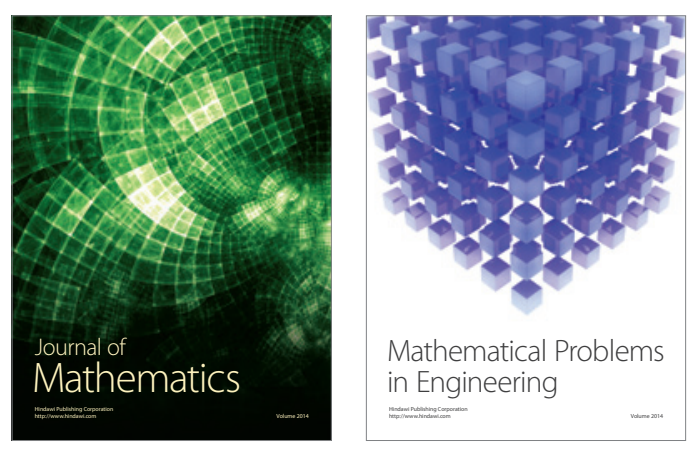

Mathematical Problems in Engineering
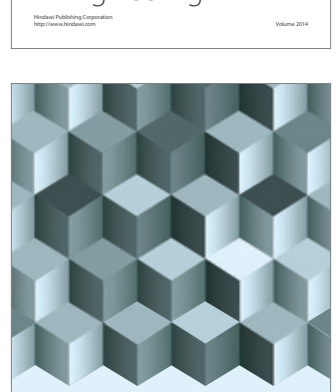

Journal of

Function Spaces
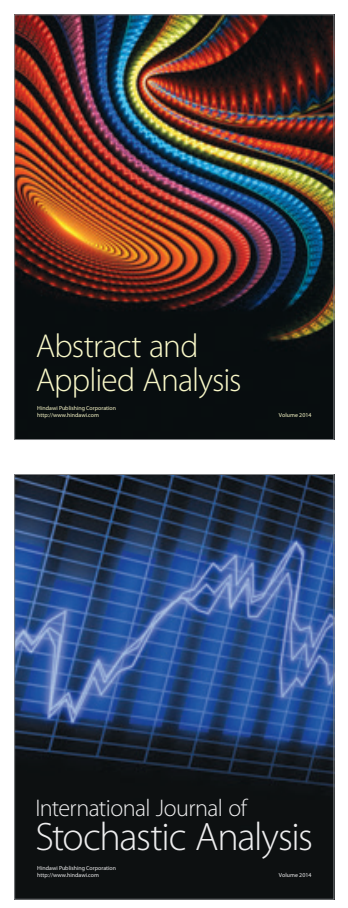

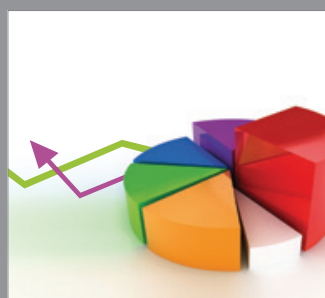

ournal of

Probability and Statistics

Promensencen
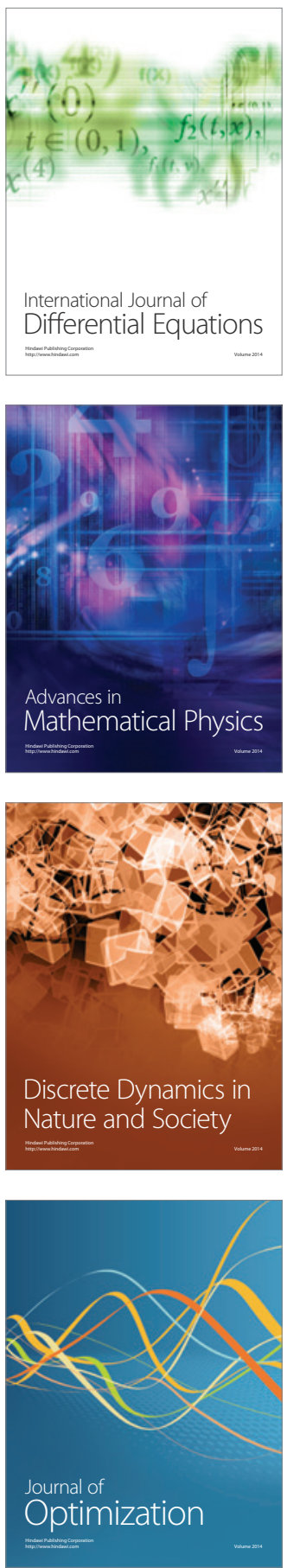